\title{
Self-sampling is appropriate for detection of Staphylococcus aureus: a validation study
}

Brigitte AGL van Cleef ${ }^{1,2,3^{*}}$, Miranda van Rijen ${ }^{3}$, Marianne Ferket $^{3}$ and Jan AJW Kluytmans $2,3,4$

\begin{abstract}
Background: Studies frequently use nasal swabs to determine Staphylococcus aureus carriage. Self-sampling would be extremely useful in an outhospital research situation, but has not been studied in a healthy population. We studied the similarity of self-samples and investigator-samples in nares and pharynxes of healthy study subjects (hospital staff) in the Netherlands.

Methods: One hundred and five nursing personnel members were sampled 4 times in random order after viewing an instruction paper: 1) nasal self-sample, 2) pharyngeal self-sample, 3) nasal investigator-sample, and 4) pharyngeal investigator-sample.

Results: For nasal samples, agreement is $93 \%$ with a kappa coefficient of 0.85 (95\% Cl 0.74-0.96), indicating excellent agreement, for pharyngeal samples agreement is $83 \%$ and the kappa coefficient is $0.60(95 \% \mathrm{Cl} 0.43-0.76)$, indicating good agreement. In both sampling sites self-samples even detected more S. aureus than investigator-samples.
\end{abstract}

Conclusions: This means that self-samples are appropriate for detection of Staphylococcus aureus and methicillin-resistant Staphylococcus aureus.

Keywords: Self-sampling, Staphylcococcus aureus, MRSA, Validation

\section{Background}

Staphylococcus aureus (S. aureus) is a well-known human commensal on skin and mucous membranes, with as most important ecological niche the nares [1,2]. In addition to colonization, $S$. aureus can cause skin and soft tissue infections, and more severe infections like necrotizing pneumonia, osteomyelitis and sepsis, which are regularly seen in hospitals in patients with other comorbidities.

Studies frequently use nasal swabs to determine S. aureus carriage, sometimes supplemented by pharyngeal swabs to increase the validity of the sampling method [3-5]. Especially in research settings in populations outside hospitals, self-sampling is often performed

\footnotetext{
*Correspondence: brigitte.van.cleef@rivm.nl

${ }^{1}$ RIVM National Institute for Public Health and the Environment, Centre for Infectious Disease Control Netherlands, P.O. Box 13720 BA, Bilthoven, the Netherlands

${ }^{2}$ St. Elisabeth hospital, Laboratory for Medical Microbiology and Immunology, Tilburg, the Netherlands

Full list of author information is available at the end of the article
}

to enhance response and lower travel costs and time for both the studied subject and the investigator [6,7].

The only study examining self-sampling for methicillinresistant S. aureus (MRSA) is Lautenbach et al. [8]. They sampled 56 MRSA-positive inpatients in the US and compared different anatomic sites and patient-collected versus provider-collected samples. Agreement percentages of $82 \%$ (nose) and 91\% (throat) were reported, demonstrating excellent agreement. However, the study population (US inpatients) is not comparable to European citizens considering health status and MRSA-prevalence; the latter is several times higher in the US compared to the Netherlands and Northern Europe $[9,10]$. Moreover, sampling order always was a sample collected by a provider, followed by a patient-collected sample. Lastly, instructions for selfsampling were not clear: were photographs available, was clearly stated which area to sample and with which technique?

We therefore studied the similarity of self-samples and investigator-samples in testing the presence of $S$. aureus in nares and pharynxes of healthy study
C Biomed Central

(c) 2012 van Cleef et al.; licensee BioMed Central Ltd. This is an Open Access article distributed under the terms of the Creative Commons Attribution License (http://creativecommons.org/licenses/by/2.0), which permits unrestricted use, distribution, and reproduction in any medium, provided the original work is properly cited. 
subjects in the Netherlands, using random sample order and clear instructions.

\section{Methods}

This crossover screening validation took place in April 2009. From nursing and technical personnel working in different wards in the Amphia hospital in Breda, the Netherlands, 4 samples were taken in random order: 1) nasal self-sample, 2) pharyngeal self-sample, 3) nasal investigator-sample, and 4) pharyngeal investigatorsample. A convenience sample size of around 100 subjects (400 samples) was determined.

Before sampling, a one page instruction was shown with photographs of both samplings (see Additional file 1), no spoken instructions were given in order to represent a true self-sample. The subject was advised to sample both inner nares, especially in the tip of the nose, and both tonsils or tonsillar arches with a different swab, both in a turning movement. Venturi Transystem swabs with Stuart medium were used (Copan Innovation, Italy). Before or after these two self-samples, the investigator sampled the nares and pharynx using the same technique as described above.

Swabs were inoculated on SA ID agar plates (bioMérieux, La Balme Les Grottes, France) and Columbia blood agar plates with 5\% sheep blood, and enriched in Mueller-Hinton broth containing 6.5\% NaCl. From the overnight Mueller-Hinton broth, $10 \mu \mathrm{l}$ was streaked onto an SA ID agar plate. The results were read after overnight incubation at $35^{\circ} \mathrm{C}$. Colonies showing green coloration were considered indicative for $S$. aureus, and were confirmed by standard techniques: colony morphology, coagulase slide test, DNase test, and a coagulase tube when discrepancies arose. Colonies with colours other than green, or no growth at all were considered negative. The procedure was performed as recommended by the manufacturer.

From $2 \times 2$ tables, percentage agreement was calculated. Cohen's simple kappa coefficients with 95\% Confidence Intervals (CI) were calculated with SAS, version 9.3 (SAS Institute Inc., Cary, NC, USA). A kappa coefficient of $>0.6$ was considered to indicate good agreement, a coefficient of $>0.8$ was considered to indicate excellent agreement, and a coefficient of 1 indicated perfect agreement. Gold standards were created combining self-samples and investigator-samples per sampling site; if either one was positive, that person was considered S. aureus positive on that site. Sensitivities were calculated with OpenEpi [11].

\section{Results}

A total of 105 nursing and technical personnel members were sampled. Thirty percent (31/105) of nasal investigatorsamples were $S$. aureus positive, compared to $34 \%(36 / 105)$ of self-samples. For pharyngeal samples, these numbers were $27 \%(28 / 105)$ and $34 \%(36 / 105)$, respectively. Table 1 shows that nasal self-samples and investigator-samples have an agreement of 93\% and a kappa coefficient of 0.85 (CI 0.74-0.96). For pharyngeal samples an agreement of $83 \%$ and a kappa coefficient of 0.60 (CI $0.43-0.76$ ) was obtained. In both sampling sites self-samples even dectected more $S$. aureus than investigator-samples.

Table 2 shows that, compared to the site-specific gold standard, nasal self-samples have a sensitivity of $97 \%$ (CI 86-100\%), and pharyngeal self-samples have a sensitivity of $88 \%$ (CI 75-95\%).

\section{Discussion}

This experimental study shows that self-samples and investigator-samples are very similar in testing the presence of S. aureus in nares and pharynxes. For nasal samples, agreement is $93 \%$ with a kappa coefficient of 0.85 , indicating excellent agreement, for pharyngeal samples agreement is $83 \%$ and the kappa coefficient is 0.60 , indicating good agreement. This means that self-samples are appropriate for detection of S. aureus and MRSA.

Furthermore, when looking at the discordant pairs, nasal self-samples tend to yield more $S$. aureus compared to investigator-samples. A rational explanation might be that swabbing is more thoroughly done by persons themselves compared to swabbing by investigators, who press less hard, resulting in fewer microorganisms picked up by the swab and a lower prevalence of $S$. aureus. For pharyngeal swabs the same can be stated, but results are less clear. This is probably due to the fact that taking swabs from tonsils or tonsillar arches is more

Table 1 Self-samples versus investigator-samples per sampling site

\begin{tabular}{llccc}
\hline Nasal samples & & \multicolumn{2}{c}{ Investigator-samples } & \multirow{2}{*}{ Total } \\
\cline { 3 - 4 } & & SApos & SAneg & \\
\hline Self-samples & SApos & 30 & 6 & 36 \\
& SAneg & 1 & 68 & 69 \\
& Total & 31 & 74 & 105 \\
\hline Agreement & & $93 \%$ & & \\
Kappa coefficient & 0.85 & (Cl 0.74-0.96) & \\
\hline A. & & &
\end{tabular}

A. Nasal samples. SApos: S. aureus positive; SAneg: S. aureus negative; Cl: confidence interval.

\begin{tabular}{lcccc}
\hline Pharyngeal samples & \multicolumn{2}{c}{ Investigator-samples } & \multirow{2}{*}{ Total } \\
\cline { 2 - 4 } & & SApos & SAneg & \\
\hline Self-samples & SApos & 23 & 13 & 36 \\
& SAneg & 5 & 64 & 69 \\
& Total & 28 & 77 & 105 \\
\hline Agreement & $83 \%$ & & \\
Kappa coefficient & 0.60 & (Cl 0.43-0.76) & \\
\hline
\end{tabular}

B. Pharyngeal samples. SApos: S. aureus positive; SAneg: S. aureus negative; $\mathrm{Cl}$ : confidence interval. 
Table 2 Self-samples versus gold standard per sampling site

\begin{tabular}{llccc}
\hline Nasal samples & & \multicolumn{2}{c}{ Gold standard } & Total \\
\cline { 3 - 4 } & & SApos & SAneg & \\
\hline Self-samples & SApos & 36 & 0 & 36 \\
& SAneg & 1 & 68 & 69 \\
& Total & 37 & 68 & 105 \\
\hline Sensitivity & & $97 \%$ & (Cl 86-100\%) &
\end{tabular}

A. Nasal samples. SApos: S. aureus positive; SAneg: S. aureus negative; Cl: confidence interval.

\begin{tabular}{lcccc}
\hline \multirow{2}{*}{ Pharyngeal samples } & \multicolumn{2}{c}{ Gold standard } & Total \\
\cline { 3 - 4 } & & SApos & SAneg & \\
\hline Self-samples & SApos & 36 & 0 & 36 \\
& SAneg & 5 & 64 & 69 \\
& Total & 41 & 64 & 105 \\
\hline Sensitivity & & $88 \%$ & (Cl 75-95\%) &
\end{tabular}

B. Pharyngeal samples. SApos: S. aureus positive; SAneg: S. aureus negative; $\mathrm{Cl}$ : confidence interval.

complex, compared to swabs from the nares. In addition, the investigators observed that the instructions for pharyngeal samples were less well adhered to than the instructions for nasal samples.

This strengthens the conclusions from Lautenbach et al., although agreement percentages are slightly different ( $82 \%$ for nares and $91 \%$ for throat) [8]. Moreover, using the information given in this reference, kappa coefficients can be calculated, which are different from the coefficients found in this study (kappa coefficients of 0.28, CI -0.05-0.60 for nares and 0.80, CI 0.64-0.97 for throat). Possible explanations for these discrepancies can be the different populations studied (US inpatients versus healthy subjects from the Netherlands) or different methods used (strict or random sampling order, type of instructions). Hanselman et al. have used nasal self-sampling, and found $S$. aureus percentages in US teachers consistent with literature, indicating appropriateness of self-sampling [12]. More studies demonstrate the usefulness of self-samples, albeit from other anatomic sites and for other microorganisms, as human papilloma virus, group-B streptococci, respiratory viruses, and sexually transmitted diseases [7,13-15].

The validity of nasal and pharyngeal self-sampling cannot be established entirely correctly, as there is no true gold standard. However, when comparing to combined self-samples and investigator-samples per site, nasal self-samples have a sensitivity of 97\% (CI 86-100\%), and pharyngeal self-samples have a sensitivity of $88 \%$ (CI 75-95\%). Lautenbach et al. have calculated sensitivities based upon a gold standard of combined samples from nares, throat, axillae, groin and perineum, and found sensitivities of 91\% (CI 80-97\%) for nasal self-samples, and $67 \%$ (CI 53-79\%) for throat self-samples [8].
This study has three limitations. The nursing personnel members are expected to have more prior knowledge of sampling methods compared to the standard outhospital person. This might lead to an overestimation of the adequacy and validity of self-sampling. Moreover, false sampling was not checked for by, for example, placing the swab on a standard sheep blood agar, which detects whether any viable microorganisms are present on the swab. However, the investigators were standing next to the subject when sampling took place, making false sampling unlikely. In addition, S. aureus prevalence corresponds to literature, confirming adequate sampling [1]. Checking for false sampling with blood agar probably is a useful suggestion when using self-samples in a research situation, however. Lastly, three investigators were involved in taking the investigator-samples, which might lead to slightly different sampling techniques. As these investigators are all well trained on sample taking and sampling techniques were discussed beforehand, we believe the effect of this variation is negligible. Data on who took which samples was unfortunately not recorded, giving no opportunity to verify this statement.

\section{Conclusions}

In conclusion, self-sampling the nares and pharynx for the presence of $S$. aureus has an excellent and good agreement with investigator-samples, respectively. Selfsampling saves both time and costs, and enhances response rate, which can be extremely useful in outhospital study situations of e.g. community-acquired or livestock-associated MRSA.

\section{Additional file}

Additional file 1: Instruction for $S$. aureus sampling.

\section{Abbreviations}

Cl: Confidence interval; MRSA: Methicillin-resistant Staphylococcus aureus; SA: Staphylococcus aureus; S. aureus: Staphylococcus aureus; US: United States..

\section{Competing interests}

The authors declare that they have no competing interests.

\section{Authors' contributions}

BvC designed the study, gathered and interpreted data, performed statistical analysis, and drafted the manuscript. MvR participated in the design of the study, gathered data and participated in drafting the manuscript. MF participated in gathering data. JK conceived the study, particpated in coordination an interpretation of data, and helped to draft the manuscript. All authors read and aproved the final manuscript.

\section{Authors' information}

BVC, MvR and JK conduct multiple outhospital studies for methicillin-resistant S. aureus, where self-sampling is very common.

\section{Acknowledgements}

We thank the 'ARIC-PILGRIM S. aureus studies fund' for giving us the financial support needed for publication. 


\section{Author details}

'RIVM National Institute for Public Health and the Environment, Centre for Infectious Disease Control Netherlands, P.O. Box 13720 BA, Bilthoven, the Netherlands. ${ }^{2}$ St. Elisabeth hospital, Laboratory for Medical Microbiology and Immunology, Tilburg, the Netherlands. ${ }^{3}$ Amphia Hospital Breda, Laboratory for Microbiology and Infection Control, Breda, the Netherlands. ${ }^{4} \mathrm{VU}$ University medical centre, Department of Medical Microbiology and Infection Prevention, Amsterdam, the Netherlands.

Received: 17 September 2012 Accepted: 6 November 2012

Published: 8 November 2012

\section{References}

1. Kluytmans J, van Belkum A, Verbrugh H: Nasal carriage of Staphylococcus aureus: epidemiology, underlying mechanisms, and associated risks. Clin Microbiol Rev 1997, 10:505-520.

2. Wertheim HF, Melles DC, Vos MC, van Leeuwen W, van Belkum A, Verbrugh HA, Nouwen JL: The role of nasal carriage in Staphylococcus aureus infections. Lancet Infect Dis 2005, 5:751-762.

3. Mertz D, Frei R, Jaussi B, Tietz A, Stebler C, Fluckiger U, Widmer AF: Throat swabs are necessary to reliably detect carriers of Staphylococcus aureus. Clin Infect Dis 2007, 45:475-477.

4. Nilsson P, Ripa T: Staphylococcus aureus throat colonization is more frequent than colonization in the anterior nares. J Clin Microbiol 2006, 44:3334-3339

5. Uemura E, Kakinohana S, Higa N, Toma C, Nakasone N: Comparative chracterization of Staphylococcus aureus isolates from throats and noses of healthy volunteers. Jpn J Infect Dis 2004, 57:21-24.

6. van der Zalm MM, Uiterwaal CS, de Jong BM, Wilbrink B, van der Ent CK: Viral specimen collection by parents increases response rate in population-based virus studies. J Allergy Clin Immunol 2006, 117:955-956. author reply 956-957.

7. Akmatov MK, Krebs S, Preusse M, Gatzemeier A, Frischmann U, Schughart K, Pessler F: E-mail-based symptomatic surveillance combined with self-collection of nasal swabs: a new tool for acute respiratory infection epidemiology. Int J Infect Dis 2011, 15:e799-803.

8. Lautenbach E, Nachamkin I, Hu B, Fishman NO, Tolomeo P, Prasad P, Bilker WB, Zaoutis TE: Surveillance cultures for detection of methicillin-resistant Staphylococcus aureus: diagnostic yield of anatomic sites and comparison of provider- and patient-collected samples. Infect Control Hosp Epidemiol 2009, 30:380-382.

9. EARS-Net. http://ecdc.europa.eu.

10. Jarvis WR, Jarvis AA, Chinn RY: National prevalence of methicillin-resistant Staphylococcus aureus in inpatients at United States health care facilities, 2010. Am J Infect Control 2012, 40:194-200.

11. Dean AG, Sullivan KM, Soe MM: OpenEpi: Open Source Epidemiologic Statistics for Public Health, Version 2.3.1. http://www.OpenEpi.com.

12. Hanselman BA, Kruth SA, Rousseau J, Weese JS: Methicillin-resistant Staphylococcus aureus colonization in schoolteachers in Ontario. Can J Infect Dis Med Microbiol 2008, 19:405-408.

13. Dijkstra MG, Heideman DA, van Kemenade FJ, Hogewoning KJ, Hesselink AT, Verkuijten MC, van Baal WM, Boer GM, Snijders PJ, Meijer CJ: Brush-based self-sampling in combination with GP5+/6+-PCR-based hrHPV testing: high concordance with physician-taken cervical scrapes for HPV genotyping and detection of high-grade CIN. J Clin Virol 2012, 54:147-151

14. Price D, Shaw E, Howard M, Zazulak J, Waters H, Kaczorowski J: Selfsampling for group B streptococcus in women 35 to 37 weeks pregnant is accurate and acceptable: a randomized cross-over trial. J Obstet Gynaecol Can 2006, 28:1083-1088

15. Freeman AH, Bernstein KT, Kohn RP, Philip S, Rauch LM, Klausner JD: Evaluation of self-collected versus clinician-collected swabs for the detection of Chlamydia trachomatis and Neisseria gonorrhoeae pharyngeal infection among men who have sex with men. Sex Transm Dis 2011, 38:1036-1039.

doi:10.1186/2047-2994-1-34

Cite this article as: van Cleef et al: Self-sampling is appropriate for detection of Staphylococcus aureus: a validation study. Antimicrobial Resistance and Infection Control 2012 1:34.

\section{Submit your next manuscript to BioMed Central and take full advantage of:}

- Convenient online submission

- Thorough peer review

- No space constraints or color figure charges

- Immediate publication on acceptance

- Inclusion in PubMed, CAS, Scopus and Google Scholar

- Research which is freely available for redistribution 